\title{
Catalogue of high-mass X-ray binaries in the Galaxy (4th edition) ${ }^{\star}$
}

\author{
Q. Z. Liu ${ }^{1,2}$, J. van Paradijs ${ }^{2}$, and E. P. J. van den Heuvel ${ }^{2}$ \\ 1 Purple Mountain Observatory, Chinese Academy of Sciences, Nanjing 210008, PR China \\ e-mail: qliu@science.uva.nl \\ 2 Sterrenkundig Instituut "Anton Pannekoek", Universiteit van Amsterdam, Kruislaan 403, 1098 SJ Amsterdam, The Netherlands
}

Received 9 February 2006 / Accepted 18 April 2006

\begin{abstract}
We present a new edition of the catalogue of high-mass X-ray binaries in the Galaxy. The catalogue contains source name(s), coordinates, finding chart, X-ray luminosity, system parameters, and stellar parameters of the components and other characteristic properties of 114 high-mass X-ray binaries, together with a comprehensive selection of the relevant literature. The aim of this catalogue is to provide the reader with some basic information on the X-ray sources and their counterparts in other wavelength ranges $(\gamma$-rays, UV, optical, IR, radio). About $60 \%$ of the high-mass X-ray binary candidates are known or suspected Be/X-ray binaries, while $32 \%$ are supergiant/X-ray binaries. Some sources, however, are only tentatively identified as high-mass X-ray binaries on the basis of their $\mathrm{X}$-ray properties similar to the known high-mass X-ray binaries. Further identification in other wavelength bands is needed to finally determine the nature of these sources. In cases where there is some doubt about the high-mass nature of the X-ray binary this is mentioned. Literature published before 1 October 2005 has, as far as possible, been taken into account.
\end{abstract}

Key words. catalogs $-\mathrm{X}$-ray: binaries - stars: emission-line, Be - stars: binaries: general

\section{Introduction}

High-mass X-ray binaries (HMXBs) were among the very first bright X-ray sources detected and optically identified in the 1970s. The systems comprise a compact object orbiting a massive OB class star. The compact object should be either a neutron star (NS) or black hole and is a strong X-ray emitter via the accretion of matter from the OB companion. Thus, cataclysmic variables do not belong to HMXBs (for a catalogue of cataclysmic variables see Ritter \& Kolb 2003). Conventionally, HMXBs can be further divided into two subgroups: those in which the primary is a Be star (Be/X-ray binary) and those in which the primary is a supergiant ( $\mathrm{SG} / \mathrm{X}$-ray binary). One can refer to the book by Lewin \& van der Klis (2006) for a full understanding of the various aspects of HMXBs.

The majority of the known high-mass X-ray binaries are $\mathrm{Be} / \mathrm{X}$-ray systems (BeXRBs), especially those in the Magellanic Clouds (Liu et al. 2005). Meurs \& van den Heuvel (1989) predict 2000-20000 Be/X-ray binaries in the Galaxy. In Be systems, the compact object is a neutron star and is typically in a wide, moderately eccentric orbit, and it spends little time in close proximity to the dense circumstellar disk surrounding the Be companion (Coe 2000; Negueruela 2004). No black hole and Be star system has been found yet (see Zhang et al. 2004). X-ray outbursts will be expected when the compact object passes through the Be-star disk, accreting from the low-velocity and high-density wind around Be stars, and thus collectively termed Be/X-ray transients. Their X-ray spectra are usually hard. The hard X-ray spectrum, along with the transience, is an important characteristic of the Be/X-ray binaries.

* Table 1 and its references are only available in electronic form at the CDS via anonymous ftp to cdsarc.u-strasbg.fr (130.79.128.5) or via

http: //cdsweb.u-strasbg.fr/cgi-bin/qcat? J/A+A/455/1165
In the second group of HMXB systems, the compact star orbits a supergiant early-type star, deep inside the highly supersonic wind (for a review see Kaper et al. 2004). The X-ray luminosity is either powered by the strong stellar wind of the optical companion or Roche-lobe overflow. In a wind-fed system, accretion from the stellar wind results in a persistent X-ray luminosity of $10^{35}-10^{36} \mathrm{erg} \mathrm{s}^{-1}$, while in a Roche-lobe overflow system, matter flows via the inner Lagrangian point to an accretion disc. A much higher X-ray luminosity $\left(\sim 10^{38} \mathrm{erg} \mathrm{s}^{-1}\right)$ is produced.

Five years after the publication of the previous ( $3 \mathrm{rd}$ ) edition (Liu et al. 2000), the amount of new literature and the number of new objects to be included have again grown so much that it seems worthwhile to concentrate the information of HMXBs in the Magellanic Clouds and in the Galaxy separately. In a previous paper we published a catalogue of $128 \mathrm{HMXBs}$ in the Magellanic Clouds (Liu et al. 2005), and here we present a catalogue of HMXBs in the Galaxy. We briefly recall some of the developments that, over the past five years, have had (and still have) a major impact on this catalogue.

Due to the much increased sensitivity and spatial resolution achievable with the Chandra and the XMM-Newton X-ray observatories, as well as with the Hubble Space Telescope and large ground-based radio telescopes, more accurate positions of $\mathrm{X}$-ray binaries have been determined, resulting in the unambiguous discovery of the optical and/or IR counterpart to some X-ray sources. Moreover, the number of HMXBs in external galaxies is also rapidly increasing, e.g., the X-ray binaries in M 81 (Swartz et al. 2003) and the Antennae galaxies (Zezas et al. 2002). Most of the ultra-luminous X-ray sources in starburst and spiral galaxies (Liu \& Mirabel 2005) are believed to be HMXBs with a black hole, specifically the B0 Ib star to NGC 5204 X-1 (Liu et al. 2004), the O9I star to M 33 X-7 (Pietsch et al. 2004) and the mid-B supergiant to M101 ULX-1 (Kuntz et al. 2005). It has been proposed that the collective X-ray luminosity of high-mass 
$\mathrm{X}$-ray binaries can be used as an indicator of the star-formation rate for the host galaxy (Grimm et al. 2003).

Since its launch in 2002, INTEGRAL has been revealing hard X-ray sources that were not easily detected in earlier soft X-ray (typically $\leq 10 \mathrm{keV}$ ) observations. Hard X-rays are not easily absorbed by matter and thus are highly penetrating. Such radiation is, therefore, ideal for probing highenergy emitting sources in dense regions. INTEGRAL has been performing a regular survey of the Galactic plane and a deep exposure of the Galactic Center as part of its Core Program (Winkler et al. 2003). A group of hard X-ray sources emitting in the hard X-ray and soft $\gamma$-ray regions have been discovered in the course of the INTEGRAL observations, which are highly absorbed, i.e., with column densities higher than about $10^{23} \mathrm{~cm}^{-2}$. The X-ray, as well as the optical/IR, properties of these sources and their location in the sky suggest that they may belong to the class of high-mass X-ray binaries, some of them possibly long-period X-ray pulsars. The donors in these binaries are most probably giant or supergiant stars (see Kuulkers 2005). For the details of all INTEGRAL sources please refer to the web page of Jerome Rodriguez (http:// isdc. unige. ch/ ${ }^{\sim}$ rodrigue/html/igrsources.html).

In recent years there has been growing evidence that there is a class of X-ray binaries referred to as fast X-ray transients, characterized with $\mathrm{X}$-ray outburst durations on the order of a few hours and peak fluxes on the order of $10^{-9} \mathrm{erg} \mathrm{cm}^{-2} \mathrm{~s}^{-1}$ $(2-10 \mathrm{keV})$. They are not readily identified with other similarly active X-ray sources: magnetically active nearby stars (i.e., DY Dra, RS CVn, or pre-main sequence stars) or superbursters (e.g., Kuulkers 2004). These sources lie in the vicinity of the Galactic Center, a region extensively monitored by INTEGRAL and other satellites. The high fluxes and the lack of nearby counterparts suggest high luminosities that would indicate an X-ray binary origin. Several of these fast transients are associated with $\mathrm{OB}$ supergiants (Coe et al. 1996; Halpern et al. 2004; Negueruela et al. 2006; Smith et al. 2006; Pellizza et al. 2006), so they are suggested to be a new class of high-mass X-ray binaries (Negueruela et al. 2005).

The aim of our catalogue is to provide some basic information on the X-ray sources and their counterparts, as well as the binary properties of the system in question, and easy access to the recent literature. No attempt has been made to compile complete reference lists. Much effort has been made to avoid errors and to keep the information up to date. Nevertheless, the authors are well aware that this edition too may contain errors and may be incomplete.

\section{Description of the table}

Table 1 lists the 114 HMXB candidates in the Galaxy. The format of Table 1 is similar to the previous edition and almost the same with the HMXB catalogue in the Magellanic Clouds (Liu et al. 2000, 2005), of which the present catalogue is meant to be an update. If there is any doubt about an entry, a question mark follows the item. In the table the sources are ordered according to the right ascension of sources; part of the (mainly numerical) information on a source is arranged in six columns, below which additional information is provided for each source in the form of key words with reference numbers [in square brackets]. The columns have been arranged as follows.

In Col. 1 the first line contains the source name, with rough information on its sky location, according to the conventional source nomenclature of space experiments in which the source was detected, hhmm \pm ddd or hhmm.m \pm ddmm. Here hh, mm, and ss indicate the hours, minutes and seconds of right ascension, ddd the declination in units of 0.1 degree (in a small number of cases, the coordinates shown in the name are given with more, or fewer, digits). The prefix $\mathrm{J}$ indicates a name based on J2000 coordinates. Otherwise, 1950 coordinates were used in the name. An alternative source name is given in the second line. In the third line of Column 1, the source types are indicated with a letter code, as follows:

- P: X-ray pulsar (66);

- T: transient X-ray source (62);

- E: eclipsing system (9);

- R: radio emitting HMXBs (9);

- C: cyclotron resonance scattering feature at X-ray spectrum (16);

- U: ultra-soft X-ray spectrum (1). These sources include black-hole candidates (BHC); some "extreme ultrasoft" (EUS) source may be a white dwarf (WD) on whose surface steady nuclear burning takes place.

Column 2 contains the right ascension (RA) and declination (Dec) of the source for equinox J2000.0 in the first two lines. RA is given as hhmmss.s to an accuracy of $0.1 \mathrm{~s}$, DEC is given in ${ }^{\circ \prime \prime}$, to an accuracy of $1^{\prime \prime}$ (in a small number of cases, the coordinates are given with more, or fewer, digits). The third line gives the galactic longitude and latitude to an accuracy of $0.1^{\circ}$ (except for sources close to the galactic center, where these coordinates are given to $0.01^{\circ}$ ). A reference for the source position is given below the columnar information under "pos.". In the parentheses following the "pos.", we provide some information on the type of observation from which the source position has been derived. The following abbreviations are used: o, optical; $\mathrm{x}, \mathrm{X}$-ray; r, radio; IR, infrared. Following the type of observation, we give an indication of the accuracy of this position, in the form of equivalent ( 90 percent confidence level) error radii, but in several cases this can only be considered an approximation (e.g. when the error box is not circular). When no accuracy is quoted, it is about one arcsecond or better.

The first and second lines of Col. 3 give the names of the optical counterpart to an X-ray source. The third line contains a reference to a finding chart. An asterisk followed by a number or letter refers to the star number used in the finding chart; "star" refers to a star in the finding chart that has not been assigned a number or letter. Many optical counterparts have been indicated with a variable-star name, as given in the General Catalogue of Variable Stars and in recent name lists of variable stars as published regularly in the IAU Information Bulletin on Variable Stars, or a number in a well-known catalogue (e.g., HD, SAO, GSC, 2MASS).

The fourth column contains some photometric information on the optical counterpart. In the first line, the apparent visual magnitude, $V$, and the color indices $B-V$ and $U-B$ are listed. The second line contains the spectral type of the optical counterpart and an estimate of the interstellar reddening, $E_{B-V}$.

The fifth column lists the near-infrared magnitudes at $J$ (1.25 microns), $H$ (1.65 microns), and $K$ s (2.17 microns), detected with the Two Micron All Sky Survey (2MASS)(Skrutskie et al. 2006).

In Col. 6, the maximum X-ray flux, or the range of observed $\mathrm{X}$-ray fluxes $(2-10 \mathrm{keV}$, unless otherwise indicated), is given in units of

$$
\begin{aligned}
1 \mu \mathrm{Jy} & =10^{-29} \mathrm{erg} \mathrm{cm}^{-2} \mathrm{~s}^{-1} \mathrm{~Hz}^{-1} \\
& =2.4 \times 10^{-12} \mathrm{erg} \mathrm{cm} \mathrm{cm}^{-2} \mathrm{~s}^{-1} \mathrm{eV}^{-1}
\end{aligned}
$$


The first line in Col. 7 gives the orbital period in days. The second line contains the pulse period for X-ray pulsars, in seconds. The third line contains a reference in which the orbital and/or pulse periods were detected.

\section{Conclusions and remarks}

The current version of this catalogue provides tabulated data and references for 114 objects, including 35 newly discovered HMXBs (2 previously listed in our low-mass X-ray binary catalogue), as well as 79 "old" ones listed in the previous catalogue. Compared with the 3rd edition, the number of HMXBs in the Galaxy listed has increased by $~ 43 \%$. Among the 114 HMXB candidates, we find 39(13) confirmed(suspected) $\mathrm{Be} / \mathrm{X}$-ray binaries (another 4 dubious Be/X-ray binaries, probably a white dwarf with a Be companion), 18(11) SG/X-ray binaries, and 6 other sources with peculiar features (CI Cam, IGR J16318-4848, Cyg X-3, LS 5039, V4641 Sgr, and SS 433). The remaining 23 systems are candidates of HMXBs. No detailed information on optical companions is known.

We would like to make some remarks on several individual sources. Both KS 1947+300 and GRO J1948+32 have been listed in the 3rd edition. Swank \& Morgan (2000), however, found that the transient X-ray source KS $1947+300$ has pulsations that are identical to the pulsar GRO J1948+32, and concluded that they are the same source. Also, the ROSAT source, RX J1037.5-5647, is the only one in the Uhuru error boxes 4U 1036-56 (Motch et al. 1997), so probably having the same origin.

MXB 0656-072 was described as a soft X-ray transient during the 1975 outburst (Clark 1975) and previously catalogued as a low-mass X-ray binary. However, observations with the PCA on board the RXTE satellite in October 2003 indicated that the source is actually a hard X-ray pulsar with a spin period of $160.7 \mathrm{~s}$ (Morgan et al. 2003). Moreover, the position of the ROSAT counterpart, RX J065817.7-071228, is consistent with a variable-reddened $09.7 \mathrm{Ve}$ spectral type Be star, suggesting a Be X-ray binary nature (Pakull et al. 2003). Another source, SAX J1819.3-2525/V4641 Sgr, was previously classified as a low-mass X-ray binary. Although the accretion processes in this system are typical of those seen in LMXBs, recent spectroscopic observations yielded a mass of the compact star in the range 5.49-8.14 $M_{\odot}$, and the optical companion was found to be a B9III star (Orosz et al. 2001). Chaty et al. (2003) also showed that the stellar type of the companion star was early type between B3 and A2 and that the binary was between intermediatemass X-ray binary and HMXB. Therefore, this system is more consistent with a HMXB than a LMXB.

We also wish to emphasize that some sources listed in this catalogue are still uncertain. They should be considered with caution, in view of all the further work needed. Some sources are tentatively classified as massive X-ray binaries due to their X-ray properties, for instance, a hard X-ray transient, a fast X-ray transient, or an extremely absorbed X-ray source. No counterpart at other bands has been found or no pulse period has been detected, or both. Also, the compact object in some weak or soft X-ray sources may be a white dwarf instead of a neutron star, e.g., $1 \mathrm{H} 0556+286$, $1 \mathrm{H} 0749-600,1 \mathrm{H} 1249-637$, and $1 \mathrm{H} 1253-761$ (Torrejon \& Orr 2001 and see Table 1). If this is the case, they should be excluded from this catalogue.

IGR J12349-6434 is tentatively identified as a HMXB candidate, primarily due to an early-type star, RT Cru, in the error of the X-ray source (Masetti et al. 2005), while XTE $\mathrm{J} 1859+083$ is included simply due to the detection of X-ray pulsations (Marshall et al. 1999). Highly absorbed hard X-ray sources are suggested as being high-mass Xray binaries (see Kuulkers 2005). Two hard X-ray sources, IGR J16418-4532 and IGR J16493-4348, with column densities of about $10^{23} \mathrm{~cm}^{-2}$ (Walter et al. 2004; Markwardt et al. 2005), are also included in this catalogue. Fast X-ray transients are probably another new class of high-mass X-ray binaries with a supergiant companion (Smith et al. 2006; Negueruela et al. 2006). The two fast transients, AX J1749.1-2733 (Sakano et al. 2002, see in't Zand 2005) and XTE J1901+014 (Remillard \& Smith 2002), together with 8 other members (XTE J1739-302, IGR J17544-2619, IGR J16465-4507, AX 1845.0-0433, AX J1841.0-0536, SAX J1818.6-1703, IGR J16479-4514, and IGR J11215-5952), are included in this edition.

Finally, the following four sources are no longer listed in our catalogue. Now it is quite clear that they do not belong to HMXBs.

1E 1024.0-5732/Wack 2134 was discovered with the Einstein Observatory. Caraveo et al. (1989) suggested that a highly reddened O5 star was the most likely optical counterpart, and therefore proposed the system was a HMXB. The source was listed in the previous catalogue. Optical and X-ray data, however, favor the system containing a Wolf-Rayet star and an O-type star (Mereghetti et al. 1994; Reig 1999). The origin of the X-rays from this source is explained by the colliding-wind binary model (Reig 1999).

1E 1048.1-5937 and GS 1845-03 were listed in the previous catalogue. GS $1845-03$ is, however, probably the same as the ASCA source, AX J1845.0-0258. Both the compact star of 1E 1048.1-5937 and AX J1845.0-0258 (Torii et al. 1998; Gotthelf \& Vasisht 1998) very likely belong to the "anomalous" $\mathrm{X}$-ray pulsars, which are now thought to be related to the magnetars. Mereghetti et al. (1998) argued that if 1E 1048.1-5937 has a companion this star must be either a white dwarf or a heliumburning star instead of a main-sequence companion.

The peculiar galactic X-ray source, 4U 1954+319, has been listed in the previous editions as an HMXB. Masetti et al. (2006), however, found that the suspected field M-type giant star is indeed the counterpart of the X-ray source, based on the Chandra Observation. They suggest that $4 \mathrm{U} 1954+319$ is a wide-orbit LMXB, most likely a neutron star, accreting from the wind of an M-type giant.

V669 Cep used to be identified as the optical counterpart

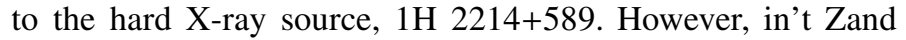
et al. (2000) identify another Be star as the optical counterpart to its BeppoSAX counterpart, SAX J2239.3+6116. Instead, V669 Cep is identified as the optical counterpart to RX J2226.6+6113 (Halpern et al. 2001). Both Hang et al. (1999) and Halpern et al. (2001) classify V669 Cep as a Herbig Ae/Be star, while Miroshnichenko et al. (2002) suggest it as a binary, consisting of a hot, low luminosity B4-B6 star and a cool companion, most likely a late-type giant. We have excluded this source in the 4th edition, since in both cases RX J2226.6+6113/V669 Cep is unlikely to be an HMXB, although it is still a HMXB in SIMBAD.

Note added in proof. After we submitted the paper, we noticed that Swift J1626.6-5156 (Negueruela et al. 2006, ATel 739), XTE J1716-389 (Cornelisse et al. 2006, MNRAS, 366, 918), IGR J16207-5129, and AX J1700.2-4220 (Palazzi et al. 2006, ATel 783) are likely candidates for high-mass X-ray binaries.

Acknowledgements. We wish to thank Marc Ribó, Sylvain Chaty and Ignacio Negueruela for carefully reading the manuscript and for their useful comments. 
We also thank Leonardo Pellizza and Sylvain Chaty for providing us with information on IGR J17544-2619 prior to publication. Finally, we are very grateful to the referees, Malcolm Coe and Sebastien Derriere, for their careful and thorough reading of this paper.

This research has made use of the SIMBAD data base operated at the CDS, Strasbourg, France, and NASA's Astrophysics Data System (ADS). This publication also makes use of data products from the Two Micron All Sky Survey, which is a joint project of the University of Massachusetts and the Infrared Processing and Analysis Center/California Institute of Technology, funded by the National Aeronautics and Space Administration and the National Science Foundation Q.Z.L. is partially supported by the Royal Science Foundation of The Netherlands, the National Natural Science Foundation of China under Grants 10173026 and 10433030, and the Major State Basic Research Development Program of China (973 Program) under Grant G1999075405.

\section{References}

Bird, A. J., Barlow, E. J., Bassani, L., et al. 2004, ApJ, 607, L33 Caraveo, P. A., Bignami, G. F., \& Goldwurm, A. 1989, ApJ, 338, 338 Chaty, S., Charles, P. A., Martí, J., et al. 2003, MNRAS, 343, 169 Clark, G. W., Schmidt, G. D., \& Angel, J. R. P. 1975, IAUC, 2843 Coe, M. J. 2000, ASPC, 214, 656

Coe, M. J. Fabregat, J., Negueruela, I., et al. 1996, MNRAS, 281, 333 Gotthelf, E. V., \& Vasisht, G. 1998, NewA, 3, 293

Grimm, H.-J., Gilfanov, M., \& Sunyaev, R. 2003, MNRAS, 339, 793 Halpern, J. P., Gotthelf, E. V., Leighly, K. M., \& Helfand, D. J. 2001, ApJ, 547,

Halpern, J. P., Gotthelf, E. V., \& Helfand, D. J. 2004, ATel, 289

Hang, H. R., Liu, Q. Z., \& Xia, J. P. 1999, A\&A, 344, 614

in't Zand, J. J. M. 2005, A\&A, 441, L1

in't Zand, J. J. M., Halpern, J., Eracleous, M., et al. 2000, A\&A, 361, 85

Kaper, L., van der Meer, A., \& Tijani, A. H. 2004, RMxAC, 21, 128

Kuntz, K. D., Gruendl, R. A., Chu, Y.-H., et al. 2005, ApJ, 620, L31

Kuulkers, E. 2004, in Proc. The Restless High-Energy Universe, ed. E. P. J. van den Heuvel, et al., Nucl. Phys. B, 132, 466

Kuulkers, E. 2005 [arXiv: astro-ph/0504625]

Lewin, W. H. G., \& van der Klis, M., ed. 2006, Compact Stellar X-Ray Sources (Cambridge University Press)
Liu, Q. Z., \& Mirabel, I. F. 2005, A\&A, 429, 1125

Liu, Q. Z., van Paradijs, J., \& van den Heuvel, E. P. J. 2000, A\&AS, 147, 25 (3rd edition)

Liu, J.-F., Bregman, J. N., \& Seitzer, P. 2004, ApJ, 602, 249

Liu, Q. Z., van Paradijs, J., \& van den Heuvel, E. P. J. 2005, A\&A, 442, 1135

Markwardt, C. B., Swank, J. H., \& Smith, E. 2005, ATel, 465

Marshall, F. E., in't Zand, J. J. M., Strohmayer, T., et al. 1999, IAUC, 7240

Masetti, N., Bassani, L., Bird, A. J., \& Bazzano, A. 2005, ATel, 528

Masetti, N., Orlandini, M., Palazzi, E., et al. 2006 [arXiv: astro-ph/0603227]

Mereghetti, S., Belloni, T., Shara, M., \& Drissen, L. 1994, ApJ, 424, 943

Mereghetti, S., Israel, G. L., \& Stella, L. 1998, MNRAS, 296, 689

Meurs, E. J. A., \& van den Heuvel, E. P. J. 1989, A\&A, 226, 88

Miroshnichenko, A. S., Bjorkman, K. S., Chentsov, E. L., et al. 2002, A\&A, 388, 563

Morgan, E., Remillard, R., \& Swank, J. 2003, ATel, 199

Motch, C., Haberl, F., Dennerl, K., et al. 1997, A\&A, 323, 853

Negueruela, I. 2004 [arXiv:astro-ph/0411335]

Negueruela, I., Smith, D. M., Reig, P., et al. 2005 [arXiv: astro-ph/0511088], in Proceedings of The X-ray Universe 2005, held in San Lorenzo de El Escorial (Madrid), 26-30 September 2005, ESA-SP 604

Negueruela, I., Smith, D. M., Reig, P., et al. 2006, ApJ, 638, 982

Orosz, J. A., Kuulkers, E., van der Klis, M., et al. 2001, ApJ, 555, 489

Pakull, M. W., Motch, C., \& Negueruela, I. 2003, ATel, 202

Pellizza, L. J., Chaty, S., \& Negueruela, I. 2006, A\&A, submitted

Pietsch, W., Mochejska, B. J., Misanovic, Z., et al. 2004, A\&A, 413, 879

Reig, P. 1999, A\&A, 345, 576

Remillard, R. A., \& Smith, D. A. 2002, ATel, 88

Ritter, H., \& Kolb, U. 2003, A\&A, 404, 301

Sakano, M., Koyama, K., Murakami, H., et al. 2002, ApJS, 138, 19

Skrutskie, M. F., Cutri, R. M., Stiening, R., et al. 2006, AJ, 131, 1163

Smith, D. M., et al. 2005, ApJ, 638, 974

Swank, J., \& Morgan, E. 2000, IAUC, 7531

Swartz, D. A., Ghosh, K. K., McCollough, M. L., et al. 2003, ApJS, 144, 213

Torii, K., Kinugasa, K., Katayama, K., et al. 1998, ApJ, 503, 843

Torrejon, J. M., \& Orr, A. 2001, A\&A, 377, 148

Walter, R., et al. 2004, HEAD, 8, 3301

Winkler, C., Courvoisier, T. J.-L., Di Cocco, G., et al. 2003, A\&A, 411, L1

Zezas, A., Fabbiano, G., Rots, A. H., \& Murray, S. S. 2002, ApJS, 142, 239

Zhang, F., Li, X.-D., \& Wang, Z.-R. 2004, ApJ, 603, 663 